\title{
FIXED-POINT THEOREMS FOR MULTIVALUED NON-EXPANSIVE MAPPINGS WITHOUT UNIFORM CONVEXITY
}

\author{
T. DOMÍNGUEZ BENAVIDES AND P. LORENZO RAMÍREZ
}

Received 13 September 2001

Let $X$ be a Banach space whose characteristic of noncompact convexity is less than 1 and satisfies the nonstrict Opial condition. Let $C$ be a bounded closed convex subset of $X, K C(C)$ the family of all compact convex subsets of $C$, and $T$ a nonexpansive mapping from $C$ into $K C(C)$. We prove that $T$ has a fixed point. The nonstrict Opial condition can be removed if, in addition, $T$ is a $1-\chi$ contractive mapping.

\section{Introduction}

Some classical fixed-point theorems for singlevalued nonexpansive mappings have been extended to multivalued mappings. The first results in this direction were established by Markin [11] in a Hilbert space setting, and by Browder [3] for spaces having a weakly continuous duality mapping. Lami Dozo [8] generalized these results to a Banach space satisfying the Opial condition.

By using Edelstein's method of asymptotic centers, Lim [9] obtained a fixedpoint theorem for a multivalued nonexpansive self-mapping in a uniformly convex Banach space. Kirk and Massa [7] gave an extension of Lim's theorem proving the existence of a fixed point in a Banach space for which the asymptotic center of a bounded sequence in a closed bounded convex subset is nonempty and compact.

Many questions remain open (see $[14,15]$ ) about the existence of fixed points for multivalued nonexpansive mappings when the Banach space satisfies geometric properties which assure the existence of a fixed point in the singlevalued case, for instance, if $X$ is a nearly uniformly convex space. In this paper, we state some fixed-point theorems for multivalued nonexpansive self-mappings, which are more general than the previous results. First, we give a fixed-point theorem for a multivalued nonexpansive and $1-\chi$-contractive mapping in the framework of a Banach space whose characteristic of noncompact convexity associated to 
the separation measure of noncompactness is less than 1. If, in addition, the space satisfies the nonstrict Opial condition, we prove, using some properties of $\chi$-minimal sets (see [2, Chapter III] for definitions), that the $\chi$-contractiveness assumption can be removed. In particular, this result gives a partial answer to [15, Problem 6].

\section{Preliminaries and notation}

Let $X$ be a Banach space. We denote by $C B(X)$ the family of all nonempty closed bounded subsets of $X$, and by $K(X)$ (resp., $K C(X)$ ) the family of all nonempty compact (resp., compact convex) subsets of $X$. On $C B(X)$ we have the Hausdorff metric $H$ given by

$$
H(A, B):=\max \left\{\sup _{a \in A} d(a, B), \sup _{b \in B} d(b, A)\right\}, \quad A, B \in C B(X),
$$

where for $x \in X$ and $E \subset X, d(x, E):=\inf \{d(x, y): y \in E\}$ is the distance from the point $x$ to the subset $E$.

If $C$ is a closed convex subset of $X$, then a multivalued mapping $T: C \rightarrow$ $C B(X)$ is said to be a contraction if there exists a constant $k \in[0,1)$ such that

$$
H(T x, T y) \leq k\|x-y\|, \quad x, y \in C,
$$

and $T$ is said to be nonexpansive if

$$
H(T x, T y) \leq\|x-y\|, \quad x, y \in C .
$$

Recall that the Kuratowski and Hausdorff measures of noncompactness of a nonempty bounded subset $B$ of $X$ are respectively defined as the numbers

$\alpha(B)=\inf \{d>0: B$ can be covered by finitely many sets of diameter $\leq d\}$,

$\chi(B)=\inf \{d>0: B$ can be covered by finitely many balls of radius $\leq d\}$.

Then a multivalued mapping $T: C \rightarrow 2^{X}$ is called $\gamma$-condensing (resp., $1-\gamma$ contractive) where $\gamma=\alpha(\cdot)$ or $\chi(\cdot)$ if, for each bounded subset $B$ of $C$ with $\gamma(B)>0$, there holds the inequality

$$
\gamma(T(B))<\gamma(B) \quad(\text { resp., } \gamma(T(B)) \leq \gamma(B)) .
$$

Here $T(B)=\cup_{x \in B} T x$. Note that a multivalued mapping $T: C \rightarrow 2^{X}$ is said to be upper semicontinuous on $C$ if $\{x \in C: T x \subset V\}$ is open in $C$ whenever $V \subset X$ is open; $T$ is said to be lower semicontinuous if $T^{-1}(V):=\{x \in C: T x \cap V \neq \varnothing\}$ is open in $C$ whenever $V \subset X$ is open; and $T$ is said to be continuous if it is both upper and lower semicontinuous. There is another different kind of continuity for set-valued operators: $T: X \rightarrow C B(X)$ is said to be continuous on $X$ (with 
respect to the Hausdorff metric $H)$ if $H\left(T x_{n}, T x\right) \rightarrow 0$ whenever $x_{n} \rightarrow x$. It is not hard to see (see $[1,4])$ that both definitions of continuity are equivalent if $T x$ is compact for every $x \in X$. We say that $x \in C$ is a fixed point of $T$ if and only if $x$ is contained in $T x$.

In the next section, we will use the following result for multivalued mappings (see also [13]).

Theorem 2.1 (see [5]). Let $X$ be a Banach space and $\varnothing \neq D \subset X$ be closed bounded convex. Let $F: D \rightarrow 2^{X}$ be upper semicontinuous $\gamma$-condensing with closed convex values, where $\gamma(\cdot)=\alpha(\cdot)$ or $\chi(\cdot)$. If $F x \cap \bar{I}_{D}(x) \neq \varnothing$ on $D$ then $\operatorname{Fix}(F) \neq \varnothing$. (Here $I_{D}(x)$ is called the inward set at $x$ defined by $I_{D}(x):=\{x+\lambda(y-x): \lambda \geq 0, y \in$ $D\}$.)

We recall some definitions of properties satisfied by a Banach space $X$.

Definition 2.2. (a) $X$ is said to be nearly uniformly convex (NUC) if it is reflexive, and its norm is uniformly Kadec-Klee, that is, for any positive number $\epsilon$ there exists a corresponding number $\delta=\delta(\epsilon)>0$ such that for any sequence $\left\{x_{n}\right\}$

$$
\left.\begin{array}{c}
\left\|x_{n}\right\| \leq 1 \quad n=1,2, \ldots \\
w-\lim _{n} x_{n}=x \\
\})=\inf \left\{\left\|x_{n}-x_{m}\right\|: n \neq m\right\} \geq \epsilon
\end{array}\right\} \Rightarrow\|x\| \leq 1-\delta .
$$

(b) $X$ is said to satisfy the Opial condition if, whenever a sequence $\left\{x_{n}\right\}$ in $X$ converges weakly to $x$, then for $y \neq x$

$$
\underset{n}{\limsup }\left\|x_{n}-x\right\|<\limsup _{n}\left\|x_{n}-y\right\| \text {. }
$$

If the inequality is nonstrict we say that $X$ satisfies the nonstrict Opial condition.

\section{Asymptotic centers and moduli of noncompact convexity}

In this section, we will consider, apart from $\alpha$ and $\chi$, another measure of noncompactness. The separation measure of noncompactness of a nonempty bounded subset $B$ of $X$ is defined by

$$
\beta(B)=\sup \left\{\epsilon \text { : there exists a sequence }\left\{x_{n}\right\} \text { in } B \text { such that } \operatorname{sep}\left(\left\{x_{n}\right\}\right) \geq \epsilon\right\} \text {. }
$$

Definition 3.1. Let $X$ be a Banach space and $\phi=\alpha, \beta$ or $\chi$. The modulus of noncompact convexity associated to $\phi$ is defined in the following way:

$$
\Delta_{X, \phi}(\epsilon)=\inf \left\{1-d(0, A): A \subset B_{X} \text { is convex, } \phi(A) \geq \epsilon\right\},
$$

where $B_{X}$ is the unit ball of $X$. 
The characteristic of noncompact convexity of $X$ associated with the measure of noncompactness $\phi$ is defined by

$$
\epsilon_{\phi}(X)=\sup \left\{\epsilon \geq 0: \Delta_{X, \phi}(\epsilon)=0\right\} .
$$

The following relationships among the different moduli are easy to obtain

$$
\Delta_{X, \alpha}(\epsilon) \leq \Delta_{X, \beta}(\epsilon) \leq \Delta_{X, \chi}(\epsilon)
$$

and consequently

$$
\epsilon_{\alpha}(X) \geq \epsilon_{\beta}(X) \geq \epsilon_{\chi}(X)
$$

When $X$ is a reflexive Banach space we have some alternative expressions for the moduli of noncompact convexity associated with $\beta$ and $\chi$,

$$
\begin{aligned}
& \Delta_{X, \beta}(\epsilon)=\inf \left\{1-\|x\|:\left\{x_{n}\right\} \subset B_{X}, x=w-\lim _{n} x_{n}, \operatorname{sep}\left(\left\{x_{n}\right\}\right) \geq \epsilon\right\}, \\
& \Delta_{X, \chi}(\epsilon)=\inf \left\{1-\|x\|:\left\{x_{n}\right\} \subset B_{X}, x=w-\lim _{n} x_{n}, \chi\left(\left\{x_{n}\right\}\right) \geq \epsilon\right\} .
\end{aligned}
$$

It is known that $X$ is NUC if and only if $\epsilon_{\phi}(X)=0$, where $\phi$ is $\alpha, \beta$ or $\chi$. The above mentioned definitions and properties can be found in [2].

Let $C$ be a nonempty bounded closed subset of $X$, and $\left\{x_{n}\right\}$ a bounded sequence in $X$, we use $r\left(C,\left\{x_{n}\right\}\right)$ and $A\left(C,\left\{x_{n}\right\}\right)$ to denote the asymptotic radius and the asymptotic center of $\left\{x_{n}\right\}$ in $C$, that is,

$$
\begin{aligned}
& r\left(C,\left\{x_{n}\right\}\right)=\inf \left\{\underset{n}{\left.\limsup \left\|x_{n}-x\right\|: x \in C\right\},}\right. \\
& A\left(C,\left\{x_{n}\right\}\right)=\left\{x \in C: \limsup _{n}\left\|x_{n}-x\right\|=r\left(C,\left\{x_{n}\right\}\right)\right\} .
\end{aligned}
$$

It is known that $A\left(C,\left\{x_{n}\right\}\right)$ is a nonempty weakly compact convex set as $C$ is.

Next, we present a theorem which gives a connection between the asymptotic center of a sequence and the modulus of noncompact convexity and it will play a crucial role in our results. First, we recall the following notation of regularity and the lemma below.

Definition 3.2. Let $\left\{x_{n}\right\}$ and $C$ be as above. Then $\left\{x_{n}\right\}$ is called regular with respect to $C$ if $r\left(C,\left\{x_{n}\right\}\right)=r\left(C,\left\{x_{n_{i}}\right\}\right)$ for all subsequences $\left\{x_{n_{i}}\right\}$ of $\left\{x_{n}\right\}$.

Lemma 3.3 (see $[6,10]$ ). Let $\left\{x_{n}\right\}$ and $C$ be as above. Then, there always exists a subsequence of $\left\{x_{n}\right\}$ which is regular with respect to $C$.

If $D$ is a bounded subset of $X$, the Chebyshev radius of $D$ relative to $C$ is defined by

$$
r_{C}(D):=\inf \{\sup \{\|x-y\|: y \in D\}: x \in C\}
$$


Theorem 3.4. Let $C$ be a closed convex subset of a reflexive Banach space $X$, and let $\left\{x_{n}\right\}$ be a bounded sequence in $C$ which is regular with respect to $C$. Then

$$
r_{C}\left(A\left(C,\left\{x_{n}\right\}\right)\right) \leq\left(1-\Delta_{X, \beta}\left(1^{-}\right)\right) r\left(C,\left\{x_{n}\right\}\right) .
$$

Moreover, if $X$ satisfies the nonstrict Opial condition then

$$
r_{C}\left(A\left(C,\left\{x_{n}\right\}\right)\right) \leq\left(1-\Delta_{X, \chi}\left(1^{-}\right)\right) r\left(C,\left\{x_{n}\right\}\right) .
$$

Proof. Denote $r=r\left(C,\left\{x_{n}\right\}\right)$ and $A=A\left(C,\left\{x_{n}\right\}\right)$. Since $\overline{c o}\left(\left\{x_{n}\right\}\right) \subset C$ is a weakly compact set, we can find a subsequence $\left\{y_{n}\right\}$ of $\left\{x_{n}\right\}$ weakly convergent to a point $z \in C$. Without loss of generality we assume that the $\operatorname{limit}_{n \neq m} \| y_{n}-$ $y_{m} \|$ exists (see [2, Theorem III.1.5]). Since $\left\{x_{n}\right\}$ is regular with respect to $C$, $r=r\left(C,\left\{y_{n}\right\}\right)$, and then, the weakly lower semicontinuity of the norm implies

$$
r \leq \limsup _{n}\left\|y_{n}-z\right\| \leq \liminf _{m} \limsup _{n}\left\|y_{n}-y_{m}\right\|=\lim _{n \neq m}\left\|y_{n}-y_{m}\right\| .
$$

Hence $\beta\left(\left\{y_{n}\right\}\right) \geq r$.

On the other hand, if $X$ satisfies the nonstrict Opial condition, it is easy to deduce that $\chi\left(\left\{y_{n}: n \in \mathbb{N}\right\}\right)=\limsup _{n}\left\|y_{n}-z\right\|$. Indeed, for every $\epsilon>0$ there exists $n_{0} \in \mathbb{N}$ such that $\left\|y_{n}-y\right\|<\limsup _{n}\left\|y_{n}-y\right\|+\epsilon$ for all $n \geq n_{0}$, and hence $\chi\left(\left\{y_{n}: n \in \mathbb{N}\right\}\right) \leq \limsup _{n}\left\|y_{n}-y\right\|$.

Conversely, suppose that $\left\{y_{n}: n \in \mathbb{N}\right\}$ can be covered by finitely many balls with radius $r<\limsup _{n}\left\|y_{n}-y\right\|$. Consider a subsequence $\left\{z_{n}\right\}$ of $\left\{y_{n}\right\}$ such that $\lim _{n}\left\|z_{n}-z\right\|=\lim \sup _{n}\left\|y_{n}-z\right\|$. Then there exists a subsequence $\left\{z_{n_{k}}\right\}$ of $\left\{z_{n}\right\}$ contained in a ball $B(x, r)$ for some $x \in X$. Therefore we obtain

$$
\underset{k}{\limsup }\left\|z_{n_{k}}-x\right\| \leq r<\limsup _{n}\left\|y_{n}-z\right\|=\lim _{k}\left\|z_{n_{k}}-z\right\|,
$$

contradicting the fact that $X$ satisfies the nonstrict Opial condition, because $z_{n_{k}} \rightarrow z$.

Thus, in this case we have $\chi\left(\left\{y_{n}: n \in \mathbb{N}\right\}\right) \geq r$.

Assume $x$ lies in $A$. Since $r=\limsup _{n}\left\|y_{n}-x\right\|$, for every $\epsilon>0$ there exists $n_{0} \in \mathbb{N}$ such that $\left\|y_{n}-x\right\|<r+\epsilon$ for all positive integer $n$ greater than or equal to $n_{0}$. Hence, the sequence

$$
\left\{\frac{y_{n}-x}{r+\epsilon}\right\}_{n \geq n_{0}}
$$

is contained in the unit ball of $X$, converges weakly to $(z-x) /(r+\epsilon)$ and $\beta\left(\left\{\left(y_{n}-x\right) /(r+\epsilon)\right\}\right) \geq r /(r+\epsilon)$.

If $X$ satisfies the nonstrict Opial condition $X$ we also have that $\chi\left(\left\{\left(y_{n}-x\right) /\right.\right.$ $(r+\epsilon)\}) \geq r /(r+\epsilon)$. Therefore we deduce

$$
\|x-z\| \leq\left(1-\Delta_{X, \beta, \tau}\left(\frac{r}{r+\epsilon}\right)\right)(r+\epsilon),
$$


and in the second assumption

$$
\|x-z\| \leq\left(1-\Delta_{X, \chi, \tau}\left(\frac{r}{r+\epsilon}\right)\right)(r+\epsilon) .
$$

Since the last inequality is true, for every $\epsilon>0$ and for every $x \in A$, we obtain the inequalities in the statement.

Remark 3.5. It must be noted that the regularity assumption is necessary in Theorem 3.4. Consider the product space $X=\ell_{\infty}^{2} \otimes \ell_{2}$, where $\ell_{\infty}^{2}:=\left(\mathbb{R}^{2},\|\cdot\|_{\infty}\right)$, with the norm

$$
\|(x, y)\|=\left(\|x\|_{\infty}^{2}+\|y\|_{2}^{2}\right)^{1 / 2}, \quad x \in \ell_{\infty}^{2}, y \in \ell_{2} .
$$

First, we are going to prove that

$$
\Delta_{X, \alpha}(\epsilon)=1-\sqrt{1-\frac{\epsilon^{2}}{4}} .
$$

Since $X$ contains isometrically $\ell_{2}$, it is easy to deduce that

$$
\Delta_{X, \alpha}(\epsilon) \leq \Delta_{\ell_{2}, \alpha}(\epsilon)=1-\sqrt{1-\frac{\epsilon^{2}}{4}},
$$

(see [2, Chapter I]).

Now, we study the reverse inequality. Taking in mind that $\alpha(A) \leq 2 \chi(A)$ (see [2]) for each bounded subset of $X$, it is clear that

$$
\Delta_{X, \alpha}(\epsilon) \geq \Delta_{X, \chi}\left(\frac{\epsilon}{2}\right)
$$

for all $\epsilon>0$. Estimate the value of $\Delta_{X, \chi}(\epsilon / 2)$. Since $X$ is reflexive, we have (see [2, Chapter V])

$$
\Delta_{X, \chi}\left(\frac{\epsilon}{2}\right)=\inf \left\{1-\|z\|: w-\lim _{n} z_{n}=z,\left\|z_{n}\right\| \leq 1, \chi\left(\left\{z_{n}\right\}\right) \geq \frac{\epsilon}{2}\right\} .
$$

Let $\left\{\left(x_{n}, y_{n}\right)\right\}$ be a sequence in the unit ball of $X$ weakly convergent to a vector $\left(x_{0}, y_{0}\right) \in X$ such that $\chi\left(\left\{\left(x_{n}, y_{n}\right)\right\}\right) \geq \epsilon / 2$.

It follows that $\lim _{n} x_{n}=x_{0}$ and $\left\{y_{n}\right\}$ is weakly convergent to $y_{0}$ in $\ell_{2}$. Taking a subsequence, if necessary, we can assume that $\lim _{n}\left\|y_{n}-y_{0}\right\|_{2}$ and $\lim _{n}\left\|y_{n}\right\|_{2}$ exist, and the supports of $y_{n}-y_{0}$ and $y_{0}$ are nearly disjoint, that is,

$$
\lim _{n}\left\|y_{n}\right\|_{2}^{2}=\left\|y_{0}\right\|_{2}^{2}+\lim _{n}\left\|y_{n}-y_{0}\right\|_{2}^{2} \text {. }
$$

On the other hand, it is not difficult to see that $X$ satisfies the Opial condition. In fact, it satisfies the uniform Opial condition with the same modulus of Opial 
associated with $\ell_{2}$. Then

$$
\chi\left(\left\{\left(x_{n}, y_{n}\right)\right\}\right)=\limsup _{n}\left\|\left(x_{n}, y_{n}\right)-\left(x_{0}, y_{0}\right)\right\|=\lim _{n}\left\|y_{n}-y_{0}\right\|_{2} \geq \frac{\epsilon}{2} .
$$

Hence

$$
\begin{aligned}
1 \geq \lim _{n}\left\|\left(x_{n}, y_{n}\right)\right\|^{2} & =\lim _{n}\left\|x_{n}\right\|_{\infty}^{2}+\left\|y_{n}\right\|_{2}^{2} \\
& =\left\|x_{0}\right\|_{\infty}^{2}+\left\|y_{0}\right\|_{2}^{2}+\lim _{n}\left\|y_{n}-y_{0}\right\|_{2}^{2} \\
& =\left\|\left(x_{0}, y_{0}\right)\right\|^{2}+\lim _{n}\left\|y_{n}-y_{0}\right\|_{2}^{2} \\
& \geq\left\|\left(x_{0}, y_{0}\right)\right\|^{2}+\frac{\epsilon^{2}}{4} .
\end{aligned}
$$

Thus

$$
\Delta_{X, \chi}\left(\frac{\epsilon}{2}\right) \geq 1-\sqrt{1-\frac{\epsilon^{2}}{4}},
$$

following the required inequality.

Moreover, since $X$ is reflexive and satisfies the uniform Opial condition then $\Delta_{X, \chi}\left(1^{-}\right)=1$ (see $[2$, Chapter V] for details).

If $x_{n} \in \mathbb{R}^{2}$ is the sequence defined by $x_{2 n-1}=(-1,0)$ and $x_{2 n}=(1,0)$ for each $n \in \mathbb{N}$, we consider the sequence $z_{n}=\left(x_{n}, 0\right) \in X$.

Denote $B$ the unit ball of $\ell_{\infty}^{2}$ and let $C=B \times\{0\}$. Clearly $C$ is a weakly compact convex subset of $X$ which contains $\left\{z_{n}\right\}$. It is not difficult to see that $r\left(C,\left\{z_{n}\right\}\right)=1$ and $A\left(C,\left\{z_{n}\right\}\right)=\{((0, y), 0): y \in[-1,1]\}$. Then $r_{C}\left(A\left(C,\left\{z_{n}\right\}\right)\right)=$ 1 , while $1-\Delta_{X, \alpha}\left(1^{-}\right)=\sqrt{3} / 2$ and $1-\Delta_{X, \chi}\left(1^{-}\right)=0$ are less than one.

\section{Main results}

In order to prove our first result, we need the following proposition which is proved along the proof of the Kirk-Massa theorem as it appears in [15].

Proposition 4.1. Let $C$ be a nonempty weakly compact separable subset of a $\mathrm{Ba}$ nach space $X, T: C \rightarrow K(C)$ a nonexpansive mapping, and $\left\{x_{n}\right\}$ a sequence in $C$ such that $\lim _{n} d\left(x_{n}, T x_{n}\right)=0$. Then, there exists a subsequence $\left\{z_{n}\right\}$ of $\left\{x_{n}\right\}$ such that

$$
T x \cap A \neq \varnothing, \quad \forall x \in A:=A\left(C,\left\{z_{n}\right\}\right) .
$$

Assume that $C$ is a nonempty weakly compact convex subset of a Banach space $X$, and $T: C \rightarrow K C(C)$ is a nonexpansive and 1- $\chi$-contractive self-mapping. Consider a bounded sequence $\left\{x_{n}\right\}$ in $C$ such that $T$ satisfies the condition

$$
T x \cap A \neq \varnothing, \quad \forall x \in A:=A\left(C,\left\{x_{n}\right\}\right) .
$$


For a fixed element $x_{0} \in A$ and an arbitrary $\mu \in(0,1]$, the contraction $T_{\mu}$ : $A \rightarrow K C(C)$ defined by

$$
T_{\mu} x=\mu x_{0}+(1-\mu) T x, \quad x \in A
$$

verifies the hypotheses of Theorem 2.1. Let $B$ be a bounded and nonprecompact subset of $C$. Since $T$ is $1-\chi$-contractive and $T_{\mu}(B)=\mu x_{0}+(1-\mu) T(B)$ we have

$$
\begin{aligned}
\chi\left(T_{\mu}(B)\right) & =\chi\left(\mu x_{0}+(1-\mu) T(B)\right)=\chi((1-\mu) T(B)) \\
& =(1-\mu) \chi(T(B)) \leq(1-\mu) \chi(B)<\chi(B) .
\end{aligned}
$$

Thus $T_{\mu}$ is $\chi$-condensing. Moreover, since $A$ is convex, $T_{\mu}$ satisfies the same boundary condition as $T$ does, that is, we have

$$
T_{\mu} x \cap A \neq \varnothing, \quad \forall x \in A .
$$

Hence by Theorem 2.1, $T_{\mu}$ has a fixed point $z_{\mu} \in A$ and we can find a sequence $\left\{z_{n}\right\}$ in $A$ satisfying $\lim _{n} d\left(z_{n}, T z_{n}\right)=0$. Notice that this conclusion is true for every $A$ closed bounded convex subset of $C$ satisfying $T x \cap A \neq \varnothing$, for all $x \in A$.

With this observation, we are able to prove our main result.

Theorem 4.2. Let $C$ be a nonempty closed bounded convex subset of a Banach space $X$ such that $\epsilon_{\beta}(X)<1$, and $T: C \rightarrow K C(C)$ be a nonexpansive and 1- $\chi$ contractive nonexpansive mapping. Then $T$ has a fixed point.

Proof. Let $x_{0} \in C$ be fixed and, for each $n \geq 1$, define

$$
T_{n} x:=\frac{1}{n} x_{0}+\left(1-\frac{1}{n}\right) T x, \quad x \in C .
$$

Then $T_{n}$ is a multivalued contraction and hence has a fixed point $x_{n}$. It is easily seen that $\lim _{n} d\left(x_{n}, T x_{n}\right)=0$. By Lemma 3.3, we may assume that $\left\{x_{n}\right\}$ is regular with respect to $C$ and using Proposition 4.1 we can also assume that

$$
T x \cap A \neq \varnothing, \quad \forall x \in A:=A\left(C,\left\{x_{n}\right\}\right) .
$$

Since condition $\epsilon_{\beta}(X)<1$ implies reflexivity [2], we apply Theorem 3.4 to obtain

$$
r_{C}(A) \leq \lambda r\left(C,\left\{x_{n}\right\}\right)
$$

where $\lambda:=1-\Delta_{X, \beta}\left(1^{-}\right)<1$.

According to the previous observation before Theorem 4.2, we can take a sequence $\left\{x_{n}^{1}\right\}$ in $A$ satisfying $\lim _{n} d\left(x_{n}^{1}, T x_{n}^{1}\right)=0$ and again reasoning as above we can assume that $\left\{x_{n}^{1}\right\}$ is regular with respect to $C$, and

$$
T x \cap A^{1} \neq \varnothing, \quad \forall x \in A^{1}:=A\left(C,\left\{x_{n}^{1}\right\}\right) .
$$


Again, applying Theorem 3.4, we obtain

$$
r_{C}\left(A^{1}\right) \leq \lambda r\left(C,\left\{x_{n}^{1}\right\}\right) .
$$

On the other hand, since $\left\{x_{n}^{1}\right\} \subset A$

$$
r\left(C,\left\{x_{n}^{1}\right\}\right) \leq r_{C}(A)
$$

and then

$$
r_{C}\left(A^{1}\right) \leq \lambda r_{C}(A)
$$

By induction, for each $m \geq 1$ we construct $A^{m}$ and $\left\{x_{n}^{m}\right\}_{n}$ where $A^{m}=$ $A\left(C,\left\{x_{n}^{m}\right\}\right),\left\{x_{n}^{m}\right\}_{n} \subset A^{m-1}$ such that $\lim _{n} d\left(x_{n}^{m}, T x_{n}^{m}\right)=0$, and

$$
r_{C}\left(A^{m}\right) \leq \lambda^{m} r_{C}(A) .
$$

Choose $x_{m} \in A^{m}$. We will prove that $\left\{x_{m}\right\}_{m}$ is a Cauchy sequence. For each $m \geq 1$ we have for any positive integer $n$

$$
\left\|x_{m-1}-x_{m}\right\| \leq\left\|x_{m-1}-x_{n}^{m}\right\|+\left\|x_{n}^{m}-x_{m}\right\| \leq \operatorname{diam} A^{m-1}+\left\|x_{n}^{m}-x_{m}\right\| .
$$

Taking upper limit as $n \rightarrow \infty$

$$
\begin{aligned}
\left\|x_{m-1}-x_{m}\right\| & \leq \operatorname{diam} A^{m-1}+\underset{n}{\limsup }\left\|x_{n}^{m}-x_{m}\right\|=\operatorname{diam} A^{m-1}+r\left(C,\left\{x_{n}^{m}\right\}\right) \\
& \leq \operatorname{diam} A^{m-1}+r_{C}\left(A^{m-1}\right) \\
& \leq 2 r_{C}\left(A^{m-1}\right)+r_{C}\left(A^{m-1}\right)=3 r_{C}\left(A^{m-1}\right) \\
& \leq 3 \lambda^{m-1} r_{C}(A) .
\end{aligned}
$$

Since $\lambda<1$, we conclude that there exists $x \in C$ such that $x_{m}$ converges to $x$. Let $x$ be a fixed point of $T$. For each $m \geq 1$,

$$
\begin{aligned}
d\left(x_{m}, T x_{m}\right) & \leq\left\|x_{m}-x_{n}^{m}\right\|+d\left(x_{n}^{m}, T x_{n}^{m}\right)+H\left(T x_{n}^{m}, T x_{m}\right) \\
& \leq 2\left\|x_{m}-x_{n}^{m}\right\|+d\left(x_{n}^{m}, T x_{n}^{m}\right)
\end{aligned}
$$

Taking upper limit as $n \rightarrow \infty$

$$
d\left(x_{m}, T x_{m}\right) \leq 2 \limsup _{n}\left\|x_{m}-x_{n}^{m}\right\| \leq 2 \lambda^{m-1} r_{C}(A) .
$$

Finally, taking limit in $m$ in both sides we obtain $\lim _{m} d\left(x_{m}, T x_{m}\right)=0$, and the continuity of $T$ implies that $d(x, T x)=0$, that is, $x \in T x$.

Remark 4.3. The inductive construction of the sequence $\left\{A^{m}\right\}_{m}$ in Theorem 4.2, also appears in [16, Theorem 3.2], though only two steps are done. 
Remark 4.4. Note that Theorem 4.2 does not hold if nonexpansiveness assumption is removed. Indeed, if $B_{2}$ is the closed unit ball of $l_{2}$ and $T: B_{2} \rightarrow B_{2}$ is defined by

$$
T(x)=T\left(x_{1}, x_{2}, \ldots\right)=\left(\sqrt{1-\|x\|^{2}}, x_{1}, x_{2}, \ldots\right)
$$

then $T$ is an $1-\chi$-contraction without a fixed point.

We do not know if $\chi$-contractiveness condition can be dropped in Theorem 4.2. In fact, it is an open problem if every nonexpansive mapping $T: C \rightarrow K C(C)$ is $1-\chi$-contractive even for single valued mappings. However, if $X$ is either a separable or a reflexive Banach space and satisfies the nonstrict Opial condition this assertion is true, as we prove in the next theorem.

THEOREM 4.5. If $X$ is either a separable or reflexive Banach space satisfying the nonstrict Opial condition, $C$ is a nonempty weakly compact subset of $X$ and $T$ : $C \rightarrow K(C)$ is a nonexpansive mapping, then $T$ is $1-\chi$-contractive.

Proof. Let $B$ be an infinite subset of $C$. Since $T(B)$ is an infinite and bounded set there exists a sequence $\left\{y_{n}\right\} \subset T(B)$ which is $\chi$-minimal (see [2, Chapter III] for definitions and properties concerning $\chi$-minimality). Since $\chi$ is strictly minimalizable we can assume that

$$
\chi\left(\left\{y_{n}: n \in \mathbb{N}\right\}\right)=\chi(T(B)) .
$$

Since $C$ is weakly compact, there is a subsequence of $\left\{y_{n}\right\}$ which is weakly convergent to some $y \in C$. Taking a subsequence, if necessary, we can suppose that $y_{n} \rightarrow y$ and $\lim _{n}\left\|y_{n}-y\right\|$ exists. As in the proof of Theorem 3.4, we have $\chi\left(\left\{y_{n}\right.\right.$ : $n \in \mathbb{N}\})=\lim _{n}\left\|y_{n}-y\right\|$.

Choose $x_{n} \in B$ such that $y_{n} \in T x_{n}$. Taking a subsequence, if necessary, we assume that $x_{n} \rightarrow u \in C, \lim _{n}\left\|x_{n}-u\right\|$ exists, and $\chi\left(\left\{x_{n}: n \in \mathbb{N}\right\}\right)=\lim _{n}\left\|x_{n}-u\right\|$.

On the other hand, because $T$ is compact valued, we can take $u_{n} \in T u$ verifying

$$
\left\|y_{n}-u_{n}\right\|=d\left(y_{n}, T u\right) \leq H\left(T x_{n}, T u\right) \leq\left\|x_{n}-u\right\|, \quad n \geq 1 .
$$

By the compactness of $T u$, we may assume that $\left\{u_{n}\right\}$ converges (strongly) to a point $v \in T u$. It follows that

$$
\begin{aligned}
\chi(T(B)) & =\lim _{n}\left\|y_{n}-y\right\| \leq \limsup _{n}\left\|y_{n}-v\right\| \\
& =\limsup _{n}\left\|y_{n}-u_{n}\right\| \leq \lim _{n}\left\|x_{n}-u\right\| \\
& =\chi\left(\left\{x_{n}\right\}\right) \leq \chi(B),
\end{aligned}
$$

and $T$ is $1-\chi$-contractive. 
In view of this result, we deduce from Theorem 4.2 the following corollary.

Corollary 4.6. Let $X$ be a Banach space with $\epsilon_{\beta}(X)<1$ which satisfies the nonstrict Opial condition. Suppose $C$ is a nonempty weakly compact convex subset of $X$ and $T: C \rightarrow K C(C)$ is a nonexpansive mapping. Then $T$ has a fixed point.

Furthermore, the method used in the proof of Theorem 4.2, applying Theorem 3.4, may be followed to obtain the following theorem.

Theorem 4.7. Let $X$ be a Banach space with $\epsilon_{\chi}(X)<1$ which satisfies the nonstrict Opial condition. Suppose $C$ is a nonempty weakly compact convex subset of $X$ and $T: C \rightarrow K C(C)$ is a nonexpansive mapping. Then $T$ has a fixed point.

This theorem extends the Kirk-Massa theorem, in the sense that we do not need the compactness of asymptotic center of a bounded sequence with respect to a bounded closed convex subset of $X$. Next example, due to Prus [12], illustrates this fact.

Example 4.8 (see [12]). Let $X_{m}$ be the space $\ell_{2}$ renormed as follows. For $x=$ $\sum_{k=1}^{\infty} x_{k} e_{k}\left(\left\{e_{k}\right\}\right.$ denotes the standard basis in $\left.\ell_{2}\right)$ set

$$
\|x\|_{m}=\sup _{n}\left(x_{n}^{2}+\frac{1}{m+1} \sum_{k=n+1}^{\infty} x_{k}^{2}\right)^{1 / 2}, \quad m \geq 1 .
$$

Clearly $\|\cdot\|_{m}$ is equivalent to the usual norm in $\ell_{2}$. The space $X_{m}$ is NUC for each $m \geq 1$, and it is easy to check that it satisfies the nonstrict Opial condition. Thus, the conclusion of Theorem 4.7 holds for these spaces. However, by nonstrict Opial condition we have for any $x \in X_{m}$

$$
\limsup \left\|x-e_{n}\right\| \geq 1
$$

while for all $k$, $n$ with $k<n$

$$
\left\|\frac{1}{\sqrt{m+1}} e_{k}-e_{n}\right\|_{m}=1
$$

Thus we conclude

$$
A\left(X_{m},\left\{e_{n}\right\}\right) \supseteq \frac{1}{\sqrt{m+1}} \overline{\operatorname{co}}\left\{e_{n}\right\}
$$

and, in particular $A\left(X_{m},\left\{e_{n}\right\}\right)$ is not compact.

Note that we cannot apply Lami-Dozo's theorem [8] to obtain a fixed point because $X_{m}$ does not satisfy strict Opial condition.

\section{Acknowledgment}

This research is partially supported by Direccion General de Enseñanza Superior (DGES), BFM-2000 0344-C02-C01. 


\section{References}

[1] J.-P. Aubin and H. Frankowska, Set-Valued Analysis, Systems \& Control: Foundations \& Applications, vol. 2, Birkhäuser Boston, Massachusetts, 1990.

[2] J. M. Ayerbe Toledano, T. Domínguez Benavides, and G. López Acedo, Measures of Noncompactness in Metric Fixed Point Theory, Operator Theory: Advances and Applications, vol. 99, Birkhäuser Verlag, Basel, 1997.

[3] F. E. Browder, Nonlinear operators and nonlinear equations of evolution in Banach spaces, Nonlinear Functional Analysis (Proc. Sympos. Pure Math., Vol. 18, Part 2, Chicago, Ill., 1968), American Mathematical Society, Rhode Island, 1976, pp. 1308.

[4] K. Deimling, Nonlinear Functional Analysis, Springer-Verlag, Berlin, 1985.

[5] Multivalued Differential Equations, de Gruyter Studies in Nonlinear Analysis and Applications, vol. 1, Walter de Gruyter \& Co., Berlin, 1992.

[6] K. Goebel, On a fixed point theorem for multivalued nonexpansive mappings, Ann. Univ. Mariae Curie-Skłodowska Sect. A 29 (1975), 69-72.

[7] W. A. Kirk and S. Massa, Remarks on asymptotic and Chebyshev centers, Houston J. Math. 16 (1990), no. 3, 357-364.

[8] E. Lami Dozo, Multivalued nonexpansive mappings and Opial's condition, Proc. Amer. Math. Soc. 38 (1973), 286-292.

[9] T. C. Lim, A fixed point theorem for multivalued nonexpansive mappings in a uniformly convex Banach space, Bull. Amer. Math. Soc. 80 (1974), 1123-1126.

[10] , Remarks on some fixed point theorems, Proc. Amer. Math. Soc. 60 (1976), 179-182.

[11] J. T. Markin, A fixed point theorem for set valued mappings, Bull. Amer. Math. Soc. 74 (1968), 639-640.

[12] S. Prus, Banach spaces with the uniform Opial property, Nonlinear Anal. 18 (1992), no. $8,697-704$.

[13] S. Reich, Fixed points in locally convex spaces, Math. Z. 125 (1972), 17-31.

[14] - Some problems and results in fixed point theory, Topological Methods in Nonlinear Functional Analysis (Toronto, Ont., 1982), Contemp. Math., vol. 21, American Mathematical Society, Rhode Island, 1983, pp. 179-187.

[15] H.-K. Xu, Metric fixed point theory for multivalued mappings, Dissertationes Math. (Rozprawy Mat.) 389 (2000), 39.

[16] _ Multivalued nonexpansive mappings in Banach spaces, Nonlinear Anal. Ser. A: Theory Methods 43 (2001), no. 6, 693-706.

T. Domínguez Benavides: Departamento de Análisis Matemático, Facultad de Matemáticas, Universidad de Sevilla, Sevilla 41080, Spain

E-mail address: tomasd@cica.es

P. Lorenzo Ramírez: Departamento de Análisis Matemático, Facultad de Matemáticas, Universidad de Sevilla, Sevilla 41080, Spain

E-mail address: ploren@cica.es 


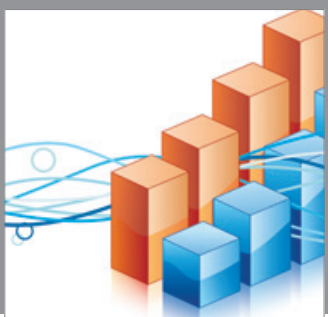

Advances in

Operations Research

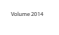

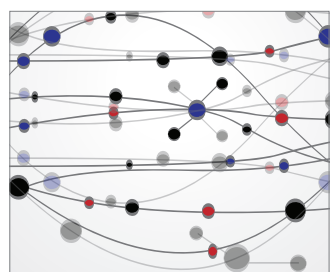

\section{The Scientific} World Journal
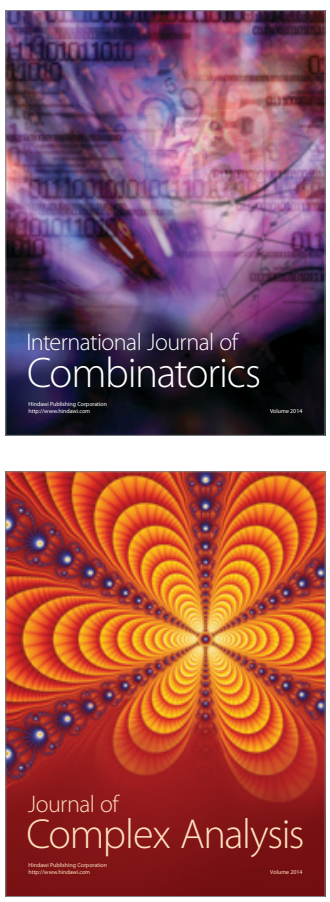

International Journal of

Mathematics and

Mathematical

Sciences
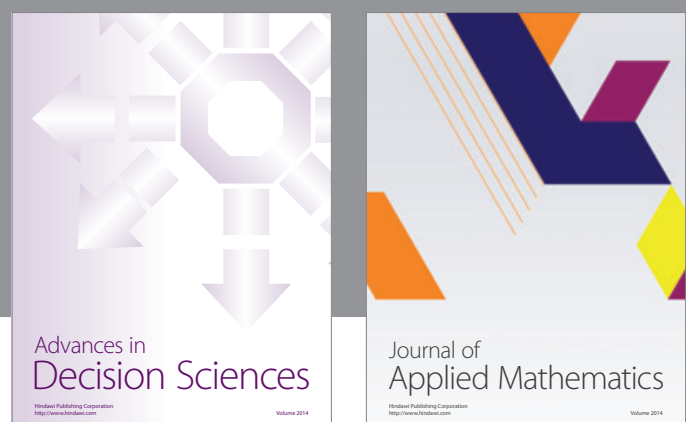

Journal of

Applied Mathematics
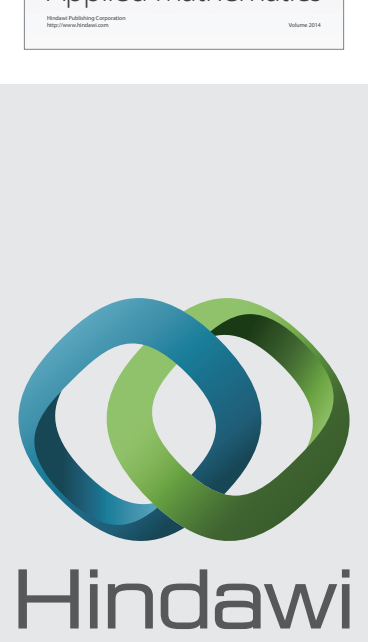

Submit your manuscripts at http://www.hindawi.com
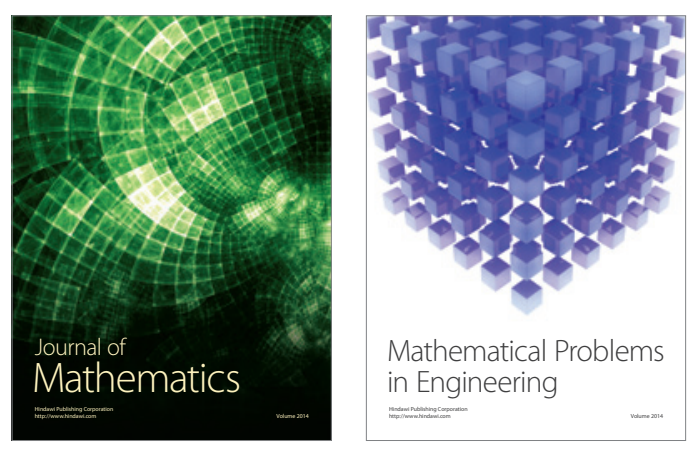

Mathematical Problems in Engineering
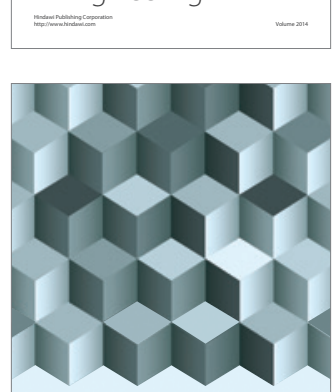

Journal of

Function Spaces
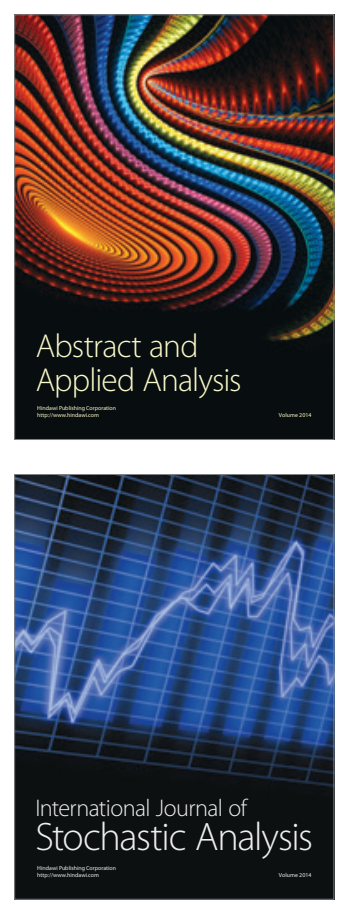

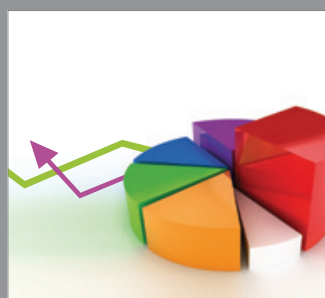

ournal of

Probability and Statistics

Promensencen
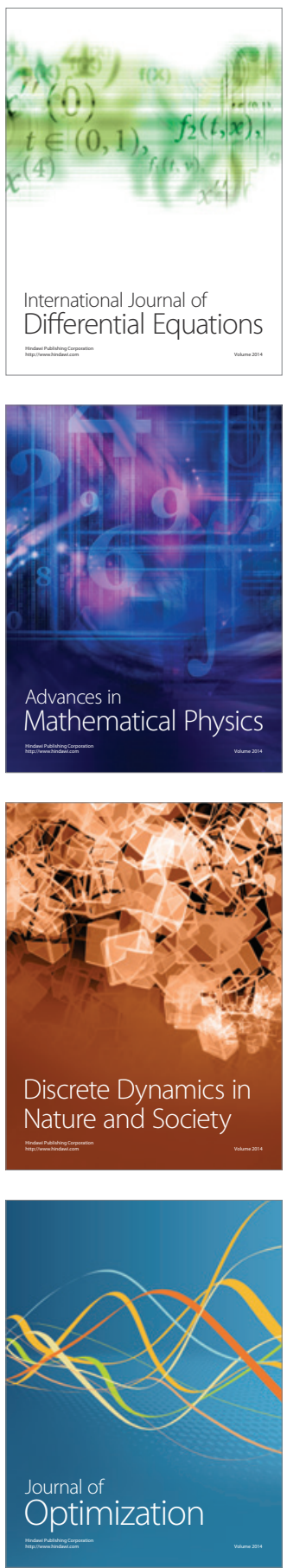